\title{
Real-space observation of spin-split molecular orbitals of adsorbed single-molecule magnets
}

Jörg Schwöbel', Yingshuang Fu', Jens Brede, Andrew Dilullo², Germar Hoffmann¹, Svetlana Klyatskaya ${ }^{3}$, Mario Ruben ${ }^{3,4} \&$ Roland Wiesendanger ${ }^{1}$

A key challenge in the field of molecular spintronics, and for the design of single-molecule magnet-based devices in particular, is the understanding and control of the molecular coupling at the electrode interfaces. It was demonstrated for the field of molecular electronics that the characterization of the molecule-metal-interface requires the precise knowledge of the atomic environment as well as the molecular orbitals being involved in electron transport. To extend the field of molecular electronics towards molecular spintronics, it is of utmost importance to resolve the spin character of molecular orbitals interacting with ferromagnetic leads. Here we present first direct real-space images of spin-split molecular orbitals of a single-molecule magnet adsorbed on a ferromagnetic nanostructure. Moreover, we are able to determine quantitatively the magnitude of the spin-splitting as well as the charge state of the adsorbed molecule.

\footnotetext{
1 Institute of Applied Physics, University of Hamburg, 20355 Hamburg, Germany. ${ }^{2}$ Department of Physics and Astronomy, Ohio University, Athens, Ohio 45701, USA. ${ }^{3}$ Institute of Nanotechnology, Karlsruhe Institute of Technology, 76344 Eggenstein-Leopoldshafen, Germany.

4 IPCMS, Université de Strasbourg, 67034 Strasbourg, France. Correspondence and requests for materials should be addressed to J.S. (email: jschwoeb@physnet.uni-hamburg.de).
} 
S ingle-molecule magnets (SMM) exhibit new properties such as electric control of molecular spin states ${ }^{1}$, remarkably high blocking temperatures, hysteresis ${ }^{2}$, quantum tunnelling of magnetization $^{3,4}$, and tunable coupling to magnetic substrates ${ }^{5}$. Recently, the realization of novel devices, such as multiplefield-effect nanotransistors ${ }^{6}$ or supramolecular spin valves ${ }^{7}$ has been reported, demonstrating the potential of SMMs for technological applications, in particular spintronics 8 and quantum computing ${ }^{9,10}$. Further progress in the field requires a detailed understanding of structural properties and magnetic interactions of an individual SMM in contact with a ferromagnetic electrode in an atomically well-defined environment. We select bis(phthalo cyaninato)terbium(III) $\left(\mathrm{TbPc}_{2}\right)$ as a model-type system because of its high blocking temperature $\left(T_{B} \sim 30-50 \mathrm{~K}\right)^{11,12}$, its comparably small size and its high stability that allows for thermal deposition in ultrahigh vacuum environments.

The structure of a $\mathrm{TbPc}_{2}$ molecule is depicted in Fig. 1a. It consists of a $\mathrm{Tb}$ ion sandwiched between two phthalocyanine (Pc) rings. The upper $\mathrm{Pc}$ ring is staggered by $45^{\circ}$ with respect to the lower plane $^{13}$, but it requires little energy to modify this twisting angle ${ }^{14}$. Neutral $\mathrm{TbPc}_{2}\left(\left[\mathrm{TbPc}_{2}\right]^{0}\right)$ contains two spin systems: a central $J=6$ Ising-type high-spin system arising from the $\mathrm{Tb} 4 \mathrm{f}$-electrons, and a peripheric $S=1 / 2 \pi$-radical delocalized over the two Pc ligands ${ }^{15}$. In neutral $\left[\mathrm{TbPc}_{2}\right]^{0}$, the highest occupied molecular orbital (HOMO), which is delocalized over both Pc rings ${ }^{15}$, is therefore just singly occupied. With spin-polarized scanning tunnelling microscopy (SP-STM), this ligand spin should be observable as follows: as the singly occupied molecular orbital of $\left[\mathrm{TbPc}_{2}\right]^{0}$ is also the lowest unoccupied molecular orbital (LUMO) ${ }^{16}$, it should be visible both below as well as above the Fermi level. Because of the Pauli exclusion principle, these orbitals have opposite spin character (Fig. 1a). Komeda et al. ${ }^{1}$ have shown that the ligand spin of $\mathrm{TbPc}_{2}$ in molecular assemblies can be switched on and off through the modification of the twisting angle. On the other hand, experimental and theoretical studies on phthalocyanine molecules show that the charge and spin state of single molecules may be altered on adsorption on surfaces ${ }^{17}$. Therefore, both the twisting and the adsorption-induced charging of $\left[\mathrm{TbPc}_{2}\right]^{0}$ molecules can cause a quenching of the ligand spin (Fig. 1b). To unambiguously identify the spin state of an adsorbed $\mathrm{TbPc}_{2}$ molecule, a direct spin-resolved study of specific a
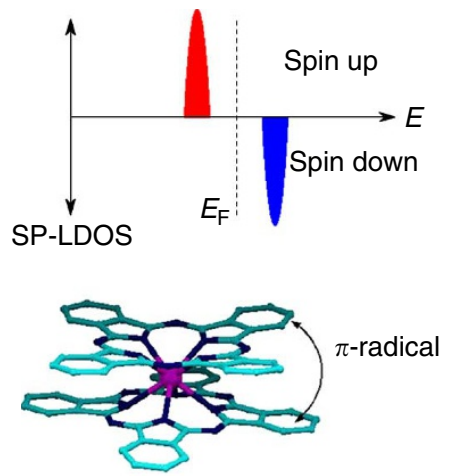

\section{b HOMO LUMO}
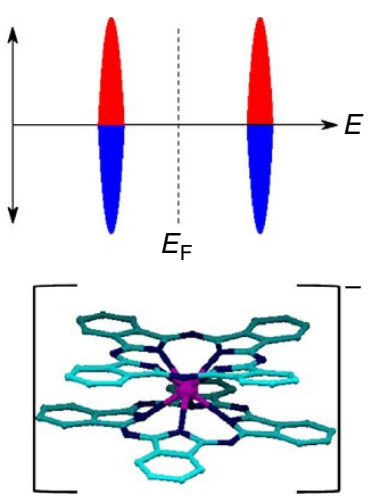

Figure 1 | Schematic spin-resolved states of $\mathrm{TbPc}_{2}$ close to the Fermi level $\left(\boldsymbol{E}_{\boldsymbol{F}}\right)$. (a) Neutral $\left[\mathrm{TbPc}_{2}\right]^{\mathrm{O}}$ : as the $\mathrm{HOMO}$ is singly occupied by a $\pi$-radical, the entire orbital is split into an occupied state below and an unoccupied state above the Fermi energy. Because of the Pauli exclusion principle, their spins are of opposite sign. (b) Negatively charged $\left[\mathrm{TbPc}_{2}\right]^{-}$: the $\pi$-radical is quenched by reduction with the additional electron. Consequently, there is no spin-split state across the Fermi level ${ }^{16}$ ligand orbitals is required. Spin-resolved investigations of simple Pc molecules on ferromagnetic substrates by SP-STM ${ }^{18,19}$ showed that the spin structure of molecule-substrate hybrid systems can indeed be mapped with high spatial and energy resolution 20,21

Here we directly observe the spin-splitting of molecular orbitals of $\mathrm{TbPc}_{2}$ molecules deposited on a ferromagnetic cobalt support. The spin polarization of individual molecules is mapped on a submolecular scale by low-temperature SP-STM, and the spin-splitting of the LUMO is determined quantitatively.

\section{Results}

Electronic structure and adsorption geometry of $\mathrm{TbPc}_{2}$. Figure $2 \mathrm{a}, \mathrm{b}$ presents STM topographs of a Co nanostructure on $\operatorname{Ir}(111)$ together with three $\mathrm{TbPc}_{2}$ molecules exhibiting three distinct orientations. The individual molecules are well separated from one another, in contrast to a similar preparation of these molecules on a $\mathrm{Au}(111)$ surface ${ }^{1}$, where the $\mathrm{TbPc}_{2}$ molecules assemble into islands. The comparison of molecular adsorption on the two different substrates indicates that molecule-substrate interactions are much stronger when $\mathrm{TbPc}_{2}$ molecules are in contact with $\mathrm{Co}$ as compared with $\mathrm{Au}$, where molecule-molecule interactions dominate. This argument is further strengthened by the observed molecular orientation discussed in Supplementary Fig. S1 and Supplementary Methods.

The spin-averaged electronic structure of $\mathrm{TbPc}_{2}$ is investigated by point spectroscopy above the ligand. The spin-averaged normalized differential tunnelling conductance $(\mathrm{d} I / \mathrm{d} U) /(I / U)$ (Fig. 2c) shows two pronounced peaks at $U=-0.9 \mathrm{~V}$ and $U=+1.3 \mathrm{~V}$. These

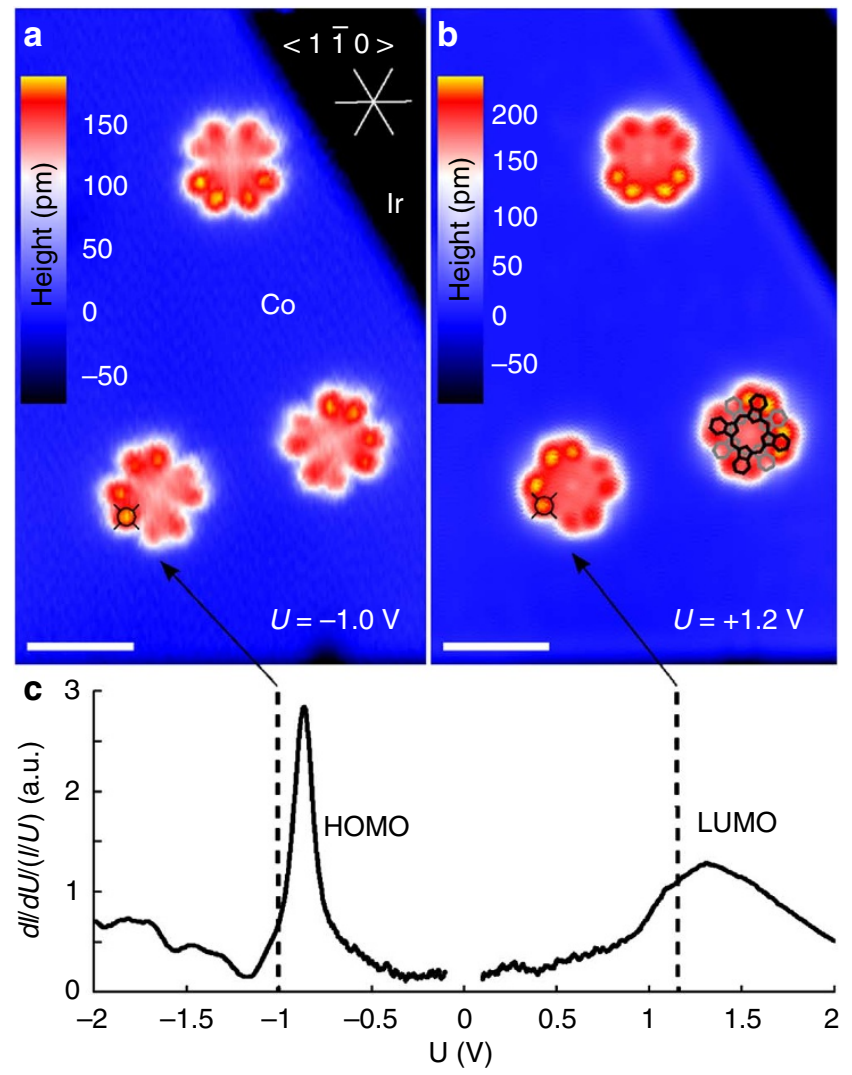

Figure 2 | Spin-averaged STM and point spectroscopy data of $\mathrm{TbPc}_{\mathbf{2}}$ on cobalt islands on Ir. (a,b) Spin-averaged STM topographs of three $\mathrm{TbPc}_{2}$ molecules on cobalt islands on Ir. The bias voltage is chosen to image either the HOMO (a) or the LUMO (b). In b, one molecule is overlaid with a model of $\mathrm{TbPc}_{2}$ with the upper Pc ring drawn in black and the lower one in grey. White scale bars correspond to $2 \mathrm{~nm}$. (c) Spin-averaged differential tunnelling spectroscopy, normalized by dividing by $I / U$. 
features are attributed to the HOMO and the LUMO, respectively. The spatial distribution of these states is imaged in the respective topographs in Fig. 2a,b and shows an eight-lobe structure for both HOMO and LUMO. This particular appearance is characteristic for $\pi$-orbitals of phthalocyanine molecules only weakly interacting with the surface ${ }^{22}$. The fact that we observe this eight-lobe structure for the $\mathrm{TbPc}_{2}$ molecules as well indicates that we are imaging the molecular orbital contribution originating from the upper Pc ring, which is only weakly electronically interacting with the Co support.

The appearance of an eight-lobe structure is expected for both a neutral $[\mathrm{TbPc} 2]^{0}$ (with the ligand spin still present) as well as for a negatively charged $\left[\mathrm{TbPc}_{2}\right]^{-}$molecule (with the ligand spin quenched by the reduction with the additional electron $)^{16}$. Therefore, spin-averaged measurements cannot distinguish between the charged and the neutral state of adsorbed $\mathrm{TbPc}_{2}$. Moreover, spin-averaged measurements cannot determine the spin state of the frontier orbitals. To address the spin character of these orbitals, SP-STM data is collected.

Spin-resolved investigation of $\mathrm{TbPc}_{2}$ molecules. Figure $3 \mathrm{a}, \mathrm{b}$ presents a scheme of SP-STM, as it is employed here: to gain spin sensitivity, a tungsten tip is coated with a ferromagnetic material (iron). $\mathrm{TbPc}_{2}$ molecules are deposited on a ferromagnetic cobalt nanostructure exhibiting a magnetization along the surface normal. To probe the two different spin channels (parallel and antiparallel), the tip magnetization (soft magnet) is aligned out of plane, either parallel or antiparallel to the magnetization of the cobalt nanostructure, by applying an external magnetic field of \pm 1 Tesla. Because of their high coercivity, the Co nanostructures act as hard magnets; therefore, their magnetization does not rotate with the external field applied here. The resulting parallel (Fig. 3a) and antiparallel (b) configurations give different tunnelling currents. The difference of these two spin channels is a measure of the spin polarization of the sample ${ }^{19}$.

First, magnetic contrast is established using the ferromagnetic Co islands as a reference (Fig. 3c-f): At $U=-0.5 \mathrm{~V}$, Co islands appear bright, when their magnetization direction is aligned parallel to the tip magnetization direction, and dark in the antiparallel case $^{23}$. $\mathrm{TbPc}_{2}$ molecules adsorbed on the ferromagnetic Co islands show a strong spin-dependent contrast as well: a clear eight-lobe state for an antiparallel and a cross-like appearance for a parallel alignment of tip and sample magnetization directions.

Spin-splitting of the LUMO. After spin contrast has been established, we focus on the analysis of the spin character of the LUMO. Figure 4 summarizes the experimental findings: the point spectroscopy mode data (Fig. 4a) show that the broad feature with a maximum at $U=+1.3 \mathrm{~V}$, which we assigned to the LUMO in the spin-averaged measurements (Fig. 2c), contains two spectroscopic features: the first feature at $U=+1.0 \mathrm{~V}$ is clearly visible only if tip and sample magnetizations are aligned antiparallel (in the following referred to as $\mathrm{LUMO}_{a p}$ ), whereas the second feature at $U=+1.2 \mathrm{~V}$ is more pronounced if tip and sample magnetizations are aligned parallel $\left(\mathrm{LUMO}_{p}\right)$. Figure $4 \mathrm{~b}$ depicts the difference of spin-resolved normalized differential tunnelling conductance for antiparallel and parallel alignment, which is a measure of the spin polarization of $\mathrm{LUMO}_{a p}$ and LUMO ${ }_{p}$. Clearly, $\mathrm{LUMO}_{a p}$ is of opposite spin character compared with $\mathrm{LUMO}_{p}$. Their exact energetic positions are determined by fitting two Lorentzian functions. The areas below the "antiparallel" and the "parallel" LUMO features are equal, indicating that the total spin polarization of the entire LUMO is zero. After determining the spin character of these states in point spectroscopy mode, we map the spatial distribution of both states in the spinsplit LUMO. To do this, we perform constant height-mode measurements. To avoid any topographic effects in SP-STM data ${ }^{24}$, the a
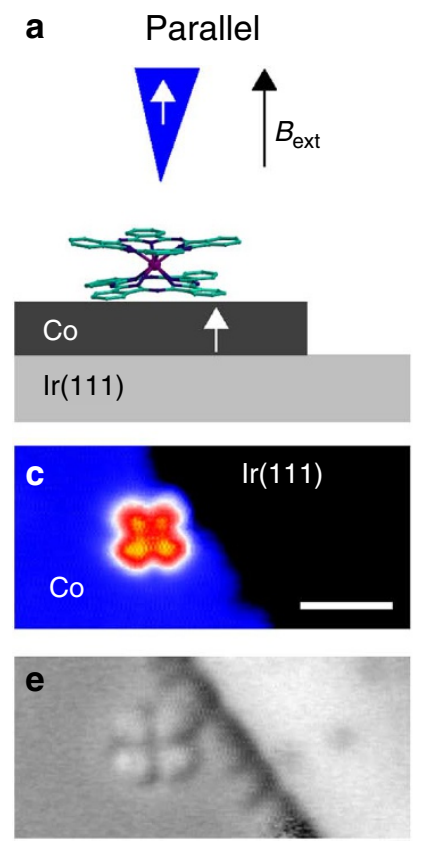

Figure 3 | Schematic diagram of SP-STM experiments as performed here. To obtain spin sensitivity, the magnetization direction of the tip is aligned either parallel (blue/dark) or antiparallel (red/light grey) by applying an external magnetic field of $B= \pm 1 \mathrm{~T}$. The Co nanostructure is a hard magnet with a magnetization that does not rotate with the field applied here. Because of the tunnel magnetoresistance effect, the tunnelling current depends on the relative alignment of tip and sample magnetization directions, thereby giving spin sensitivity. To isolate the spin-polarized component, signals from measurements with parallel (a) and antiparallel (b) alignments of tip and sample magnetization are subtracted from each other. (c-f) Example of a spin-resolved measurement: SP-STM topographs (c, parallel; d, antiparallel) and spin-resolved maps of differential conductance (e, parallel; $\mathbf{f}$, antiparallel) taken at $U=-0.5 \mathrm{~V}$. The white scale bar corresponds to $2 \mathrm{~nm}$. The topographs (c,d) show little difference, the maps of spin-resolved differential conductance $(\mathbf{e}, \mathbf{f})$ do: the Co island appears brighter for parallel alignment of tip and sample magnetization. Not only the $\mathrm{Co}$ island, but also the $\mathrm{TbPc}_{2}$ molecule shows magnetic contrast, that is, an eight-lobe structure for antiparallel and a cross-shape for parallel alignment.

tip is stabilized above the non-magnetic Ir substrate, which results in the same tip-sample distance for both parallel and antiparallel alignment of the tip and the Co-island magnetization direction. The difference between the antiparallel and parallel case yields information about the spatial distribution of the molecular spin polarization (Fig. 4c,d): for $U=+1.0 \mathrm{~V}$, it is positive, and negative for $U=+1.3 \mathrm{~V}$, consistent with the point spectroscopy data. More importantly, both orbitals are clearly of eight-lobe character. This proves that both features originate from the LUMO $\pi$-orbital. The fact that $\mathrm{LUMO}_{a p}$ and $\mathrm{LUMO}_{p}$ have an identical spatial distribution with opposite spin character allows us to unambiguously attribute the two spectral features in the different spin channels to the same molecular orbital, that is, we spatially and energetically resolve the spin-splitting of the LUMO. The magnitude of the spin-splitting of the LUMO amounts to $\Delta E=e U^{L U M O P}-e U^{L U M O a p}=210 \pm 20 \mathrm{meV}$. As shown above, the total spin polarization of the LUMO is zero, thereby excluding the presence of a radical spin in the LUMO (Fig. 4e).

\section{Discussion}

In this experiment $(T=6 \mathrm{~K})$, we do not observe any spin polarization directly arising from the central $\mathrm{Tb}$ atom. Moreover, the second 
a

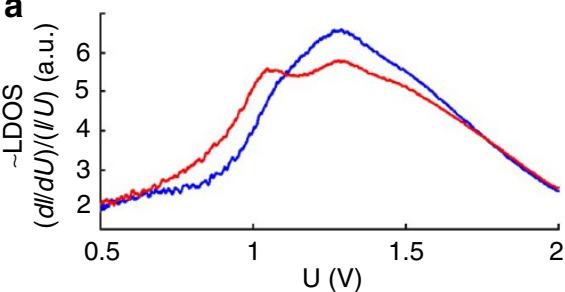

e
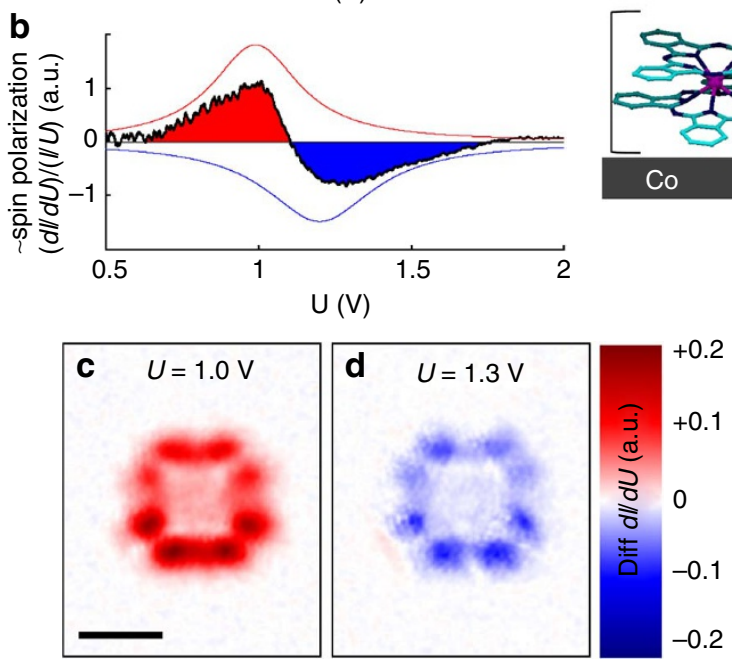

Figure 4 | Spin-resolved STM and point spectroscopy data of the LUMO. (a) Spin-resolved point-mode spectroscopy of $\mathrm{TbPc}_{2}$. Blue (dark), parallel alignment of tip and sample magnetization direction; red (light grey), antiparallel. (b) Difference of the spectroscopic curves of the antiparallel and parallel case from (a), providing a measure of the spin polarization. The area of blue (dark) and red (light grey) parts are equal, indicating that there is no net magnetization. Smooth red and blue curve: fit by two Lorentzian functions. (c,d) Differences of maps of spin-resolved differential tunnelling conductance for (c) $U=+1.0 \mathrm{~V}$ and (d) $U=+1.3 \mathrm{~V}$, providing maps of the spin polarization of the molecular orbitals at the given voltages. Both orbitals show the same spatial distribution, but have opposite spin polarization. The black scale bar corresponds to $1 \mathrm{~nm}$. (e) Schematic of the electronic structure of $\mathrm{TbPc}_{2}$ adsorbed on a cobalt nanostructure, depicted according to Fig. 1.

spin system of $\left[\mathrm{TbPc}_{2}\right]^{0}$, the $S=1 / 2$ ligand spin, is not present here, as shown above. Nevertheless, there is a spin-split on the LUMO, whose clear eight-lobe structure closely resembles a pristine molecular orbital. The spin-splitting of the LUMO can either arise owing to coupling to the ferromagnetic Co support, or be mediated by the $\mathrm{Tb}$ ion. Because the $4 \mathrm{f}$-electrons of the $\mathrm{Tb}$ ion are highly localized, a direct coupling of the $\mathrm{Tb}$ ion to the Co support is unlikely (recent studies assume a superexchange coupling mediated via the Pc ligand $\left.{ }^{5}\right)$. Additionally, calculations by Vitali et al. ${ }^{16}$ do not show a spin-splitting for the ligands for free and negatively charged $\mathrm{TbPc}_{2}$ and for molecules in contact with $\mathrm{Cu}(111)$ surfaces. Therefore, we attribute the spin-splitting of the LUMO to a direct magnetic coupling of the Co support to both Pc rings.

In conclusion, we have spatially and energetically resolved the highest occupied and the lowest unoccupied molecular orbital. Moreover, we have mapped the spatial distribution of both spincomponents of a spin-split molecular orbital on a sub-molecular level. The spin-splitting has been determined to be on the order of $210 \mathrm{meV}$. Furthermore, we conclude that the ligand spin is quenched on the upper ring upon adsorption of $\mathrm{TbPc}_{2}$ molecules on the Co nanostructures. As in ref. 1, the Tb magnetic moment was not directly accessible, raising the importance of the ligand spin system for applications in spintronic devices. The observation of a spin-split molecular orbital even in the absence of a radical spin on the ligand is a crucial result of our experimental study.

\begin{abstract}
Methods
Experimental set-up. All measurements are performed with a home-built SP-STM ${ }^{25}$ under ultrahigh vacuum conditions at a temperature of $6 \mathrm{~K}$. Magnetic fields are applied parallel or antiparallel to the surface normal. Spin-sensitive probe tips (W tips coated with $\sim 50$ monolayers of $\mathrm{Fe}$ ) and ferromagnetic cobalt nanostructures on an $\operatorname{Ir}(111)$ substrate are prepared in vacuo, according to the procedure described in ref. 23.
\end{abstract}

Sample preparation. $\mathrm{TbPc}_{2}$ molecules are synthesized, as described before ${ }^{13}$, degassed in vacuo at $\sim 300^{\circ} \mathrm{C}$ for several days, and sublimated in situ from a ceramic Knudsen cell ${ }^{26}$ held at $\sim 400^{\circ} \mathrm{C}$ directly onto the sample kept at room temperature. Following molecule deposition, the sample is directly transferred into the SP-STM system and cooled down to measurement temperature. Supplementary Fig. S2 proves that the molecules remain intact on deposition.

Additional information on the molecular adsorption, discussing moleculesubstrate interaction and twisting angle, can be found in the supplement.

Data acquisition. The differential conductance is recorded using lock-in technique with parameters given below. Point spectra are normalized by dividing by $I / U$. STM and point spectroscopy parameters:

Fig. $2 \mathrm{a}, \mathrm{b}: I=100 \mathrm{pA}$

Fig. $2 \mathrm{c}: U_{\text {stab }}=+2 \mathrm{~V}, I_{\text {stab }}=1 \mathrm{nA}, U_{\mathrm{mod}}=50 \mathrm{mV}_{\mathrm{rms}}, f_{\mathrm{mod}}=6777 \mathrm{~Hz}$

Fig. 3: $I=1 \mathrm{nA}, U_{\text {mod }}=50 \mathrm{mV}_{\text {rms }}, f_{\bmod }=6777 \mathrm{~Hz}, B= \pm 1 \mathrm{~T}$

Fig. 4a,b: Curves for both spin channels have been averaged over 32 spectra taken over all 8 lobes. $U_{\text {stab }}=+2 \mathrm{~V}, I_{\text {stab }}=1 \mathrm{nA}, U_{\bmod }=10 \mathrm{mV}_{\mathrm{rms}}, f_{\mathrm{mod}}=6777$

$\mathrm{Hz}, \mathrm{B}= \pm 1 \mathrm{~T}$

Fig. $4 \mathrm{c}$,d: $I_{\mathrm{stab}}=1 \mathrm{nA}, \Delta z_{\mathrm{stab}}=-4.5 \AA, U_{\mathrm{mod}}=50 \mathrm{mV}_{\mathrm{rms}}, f_{\mathrm{mod}}=6777 \mathrm{~Hz}, B= \pm 1 \mathrm{~T}$.

\section{References}

1. Komeda, T. et al. Observation and electric current control of a local spin in a single-molecule magnet. Nat. Commun. 2, 217 (2011).

2. Mannini, M. et al. Magnetic memory of a single-molecule quantum magnet wired to a gold surface. Nat. Mater. 8, 194-197 (2009).

3. Sessoli, R., Gatteschi, D., Caneschi, A. \& Novak, M. A. Magnetic bistability in a metal-ion cluster. Nature 365, 141-143 (1993).

4. Ishikawa, N., Sugita, M. \& Wernsdorfer, W. Quantum tunneling of magnetization in lanthanide single-molecule magnets: bis(phthalocyaninato)terbium and bis(phthalocyaninato)dysprosium Anions. Angew. Chem. Int. Ed. 44, 2931-2935 (2005).

5. Lodi Rizzini, A. et al. Coupling single molecule magnets to ferromagnetic substrates. Phys. Rev. Lett. 107, 177205 (2011).

6. Candini, A., Klyatskaya, S., Ruben, M., Wernsdorfer, W. \& Affronte, M. Graphene spintronic devices with molecular nanomagnets. Nano Lett. 11, 2634-2639 (2011).

7. Urdampilleta, M., Klyatskaya, S., Cleuziou, J.- P., Ruben, M. \& Wernsdorfer, W. Supramolecular spin valves. Nat. Mater. 10, 502-506 (2011).

8. Bogani, L. \& Wernsdorfer, W. Molecular spintronics using single-molecule magnets. Nat. Mater. 7, 179-186 (2008).

9. Dei, A. \& Gatteschi, D. Molecular (nano) magnets as test grounds of quantum mechanics. Angew. Chem. Int. Ed. 50, 11852-11858 (2011).

10. Leuenberger, M. N. \& Loss, D. Quantum computing in molecular magnets. Nature 410, 789-793 (2001).

11. Ishikawa, N., Sugita, M., Ishikawa, T., Koshihara, S.- Y. \& Kaizu, Y. Lanthanide double-decker complexes functioning as magnets at the single-molecular level. J. Am. Chem. Soc. 125, 8694-8695 (2003).

12. Branzoli, F. et al. Spin dynamics in the negatively charged terbium (iii) bis-phthalocyaninato complex. J. Am. Chem. Soc. 131, 4387-4396 (2009).

13. De Cian, A., Moussavi, M., Fischer, J. \& Weiss, R. Synthesis, structure, and spectroscopic and magnetic properties of lutetium(III) phthalocyanine derivatives: $\mathrm{LuPc}_{2} \mathrm{CH}_{2} \mathrm{Cl}_{2}$ and $\left[\mathrm{LuPc}(\mathrm{OAc})\left(\mathrm{H}_{2} \mathrm{O}\right)_{2}\right] \mathrm{H}_{2} \mathrm{O} 2 \mathrm{CH}_{3} \mathrm{OH}$. Inorg. Chem. 24, 3162-3167 (1985).

14. Qi, D. et al. Conformational effects, molecular orbitals, and reaction activities of bis(phthalocyaninato) lanthanum double-deckers: density functional theory calculations. Phys. Chem. Chem. Phys. 13, 13277-13286 (2011).

15. Ishikawa, N. et al. Upward temperature shift of the intrinsic phase lag of the magnetization of bis(phthalocyaninato)terbium by ligand oxidation creating an S = 1/2 Spin. Inorg. Chem. 43, 5498-5500 (2004).

16. Vitali, L. et al. Electronic structure of surface-supported Bis(phthalocyaninato) terbium(III) single molecular magnets. Nano Lett. 8, 3364-3368 (2008).

17. Zhao, A. et al. Controlling the Kondo effect of an adsorbed magnetic ion through its chemical bonding. Science 309, 1542-1544 (2005).

18. Wiesendanger, R., Güntherodt, H.- J., Güntherodt, G., Gambino, R. J. \& Ruf, R. Observation of vacuum tunneling of spin-polarized Electrons 
with the scanning tunneling microscope. Phys. Rev. Lett. 65, 247-250 (1990).

19. Wiesendanger, R. Spin mapping at the nanoscale and atomic scale. Rev. Mod. Phys. 81, 1495-1550 (2009).

20. Iacovita, C. et al. Visualizing the spin of individual cobalt-phthalocyanine molecules. Phys. Rev. Lett. 101, 116602 (2008).

21. Brede, J. et al. Spin- and energy-dependent tunneling through a single molecule with intramolecular spatial resolution. Phys. Rev. Lett. 105, 047204 (2010)

22. Nazin, G., Wu, S. \& Ho, W. Tunneling rates in electron transport through double-barrier molecular junctions in a scanning tunneling microscope. Proc. Natl Acad. Sci. USA 102, 8832-8837 (2005).

23. Bickel, J. E. et al. Magnetic properties of monolayer Co islands on $\operatorname{Ir}(111)$ probed by spin-resolved scanning tunneling microscopy. Phys. Rev. B 84, 054454 (2011).

24. Kubetzka, A., Pietzsch, O., Bode, M. \& Wiesendanger, R. Determining the spin polarization of surfaces by spin-polarized scanning tunneling spectroscopy. Appl. Phys. A 76, 873-877 (2003).

25. Wittneven, C., Dombrowski, R., Pan, S. H. \& Wiesendanger, R. A lowtemperature ultrahigh-vacuum scanning tunneling microscope with rotatable magnetic field. Rev. Sci. Instrum. 68, 3806-3810 (1997).

26. Lämmle, K., Schwarz, A. \& Wiesendanger, R. Miniaturized transportable evaporator for molecule deposition inside cryogenic scanning probe microscopes. Rev. Sci. Instrum. 81, 053902 (2010).

\section{Acknowledgements}

We thank N. Atodiresei for many fruitful discussions. This work is funded by the DFG via SFB668-A5 and SFB/TRR 88 3MET, the EU via the ERC Advanced Grant FURORE, the Hamburgische Stiftung für Wissenschaft und Forschung via the Cluster of Excellence NANOSPINTRONICS, and the National Science Foundation via the SPIRE project.

\section{Author contributions}

S.K. and M.R. synthesized the molecules. G.H., M.R., and R.W. planned, and J.S., Y.F., and A.D. performed the experiments. J.S. did the data analysis. J.S. and J.B. wrote the paper. All authors discussed the results and commented on the manuscript and contributed to it.

\section{Additional information}

Supplementary Information accompanies this paper at http://www.nature.com/ naturecommunications

Competing financial interests: The authors declare no competing financial interests.

Reprints and permission information is available online at http://npg.nature.com/ reprintsandpermissions/

How to cite this article: Schwöbel, J. et al. Real-space observation of spin-split molecular orbitals of adsorbed single-molecule magnets. Nat. Commun. 3:953 doi: 10.1038/ncomms1953 (2012). 